\title{
Whole-Cell Patch-Clamp Recording Reveals Subthreshold Sound-Evoked Postsynaptic Currents in the Inferior Colliculus of Awake Bats
}

\author{
Ellen Covey, Julie A. Kauer, and John H. Casseday \\ Department of Neurobiology, Duke University Medical Center, Durham, North Carolina 27710
}

The inferior colliculus receives excitatory and inhibitory input from parallel auditory pathways that differ in discharge patterns, latencies, and binaural properties. Processing in the inferior colliculus may depend on the temporal sequence in which excitatory and inhibitory synaptic inputs are activated and on the resulting balance between excitation and inhibition. To explore this issue at the cellular level, we used the novel approach of whole-cell patch-clamp recording in the midbrain of awake bats (Eptesicus fuscus) to record EPSCs or IPSCs. Sound-evoked EPSCs were recorded in most neurons. These EPSCs were frequently preceded by an IPSC, followed by an IPSC, or both. These findings help explain the large latency range and transient responses that characterize inferior colliculus neurons. The EPSC was sometimes followed by longlasting oscillatory currents, suggesting that a single brief sound sets up a pattern of altered excitability that persists far beyond the duration of the initial sound. In three binaural neurons, ipsilateral sound evoked a large IPSC that partially or totally canceled the EPSC evoked by contralateral sound. In one binaural neuron with ipsilaterally evoked IPSCs, contralaterally evoked IPSCs occurred in response to frequencies above and below the neuron's best frequency. Thus, both monaural and binaural interactions can occur at single inferior colliculus neurons. These results show that whole-cell patch-clamp recording offers a powerful means of understanding how subthreshold processes determine the responses of auditory neurons.

Key words: in vivo whole-cell patch-clamp recording; intracellular recording; inferior colliculus; auditory midbrain; postsynaptic currents; central auditory system
The inferior colliculus (IC) is one of the main integrative centers for auditory information in the mammalian brain. It provides nearly all of the input to the thalamocortical pathway, as well as direct outputs to systems concerned with sensorimotor integration. The IC is the target of at least a dozen anatomically and functionally distinct pathways, some or all of which may converge at the level of a single cell. Some input pathways are excilatory, others inhibitory. Neurons in different input pathways have different discharge patterns, and their activity occurs at different times relative to sound onset. The inputs to a single IC region or cell may differ in frequency tuning, thresholds, rate-level functions, and binaural properties. A major issue in understanding central auditory processing is how multiple synaptic inputs converge and interact to shape the response properties of IC neurons (Covey and Casseday, 1995; Casseday and Covey, 1996a,b).

There is evidence that synaptic inhibition plays a key role in the integration process. The IC contains both GABAergic and glycinergic terminals (Roberts and Ribak, 1987; Winer et al., 1995) and receptors (Glendenning and Baker, 1988; Edgar and Schwartz, 1990; Fubara et al., in press). Blocking either $\mathrm{GABA}_{\mathrm{A}}$ or glycine receptors can decrease response latency (Faingold et al., 1991;

Received Dec. 12, 1995; revised Feb. 8, 1996; accepted Feb. 12, 1996.

This work was supported by grants from the National Science Foundation (IBN9210299), the National Institute on Deafness and other Communication Disorders, National Institutes of Health (DC-00287 and DC-00607), and National Institute of Neurological Disorders and Stroke (NS30500). We thank Joan Xie and Sotirios Keros for their help in designing a system to digitize and display data obtained during whole-cell patch-clamp recording. We also thank Drs. George Augustine, William Hall, and Richard Mooney for their critical reading of this manuscript and many helpful suggestions.

Correspondence should be addressed to Ellen Covey, Department of Psychology, Box 351525, The University of Washington, Seattle, WA 98195-1525.

Copyright (C) 1996 Society for Neuroscience $0270-6474 / 96 / 163009-10 \$ 05.00 / 0$
Park and Pollak, 1993b), broaden frequency tuning curves (Yang et al., 1992), change binaural properties (Vater et al., 1992; Park and Pollak, 1993a), or change monaural properties (Pollak and Park, 1993; Casseday et al., 1994).

Although neuropharmacological methods indicate that inhibition is involved in integrative processing at the IC, we still lack direct evidence about the mechanisms that determine when an action potential occurs. It is especially important to observe processes that operate below spike threshold under normal conditions, because they provide information about the underlying dynamic balance between excitation and inhibition. The most direct way to address this issue is through intracellular recording.

To date there have been only two published studies of intracellular recordings from IC neurons in mammals, both in anesthetized cats (Nelson and Erulkar, 1963; Kuwada et al., 1980). This paucity of intracellular evidence may be because cells are often damaged when impaled with sharp electrodes (Marty and Neher, 1983). Because of the success of whole-cell patch-clamp recording in isolated or cultured cells (Hamill et al., 1981; Fenwick et al., 1982a,b; Sakmann ct al., 1982), brain slices (Blanton et al., 1989), and neurons in the visual cortex of anesthetized animals (Ferster and Jagadeesh, 1992; Jagadeesh et al., 1992, 1993), we explored this technique in the IC of awake animals. The whole-cell recording method allows long-duration recordings from healthy cells, and the low-resistance electrodes offer superior signal-to-noise properties. This report provides the first evidence that whole-cell patch-clamp recording in the midbrain of awake animals can be used to examine sensory integration. To make this point, we show how several different intracellular response properties can explain findings obtained with extracellular recording methods. 


\section{MATERIALS AND METHODS}

The methods for surgical preparation of animals and acoustic stimulation were essentially the same as those previously described in detail for extracellular recording (Covey, 1993). On the day before recording, the animal was anesthetized with a combination of Metofane (methoxyflurane) and Innovar-Vet (fentanyl, $0.4 \mathrm{mg} / \mathrm{ml}+$ droperidol, $20 \mathrm{mg} / \mathrm{ml}$; $0.125 \mathrm{ml} / \mathrm{kg}$ ). Local anesthetic (Xylocaine) was applied to the scalp. A metal post was attached to the surface of the skull with cyanoacrylate gel adhesive. When fixed in the stereotaxic apparatus with a screw, the post served to immobilize the animal's head. On the next day, before placement in the stereotaxic device, the bat was tranquilized with Innovar-Vet. During recording, local anesthetic was applied to the scalp incision.

During stimulation and recording, the bat was inside a soundattenuating chamber. The electronic apparatus for generating signals and for electrophysiological recording was outside the chamber. During the recording session, the bat was restrained in a foam-lined holder molded to the shape of the body to hold it firmly but comfortably. The holder was suspended in an elastic sling to damp movements. Water was offered at regular intervals during the recording session. If the bat showed signs of restlessness, the session was terminated. Each recording session was $\leq 6$ $\mathrm{hr}$ in duration. Between recording sessions, bats were housed in individual cages and given free access to food and water.

The IC of this species is not covered by neocortex or cerebellum, and it is visible through the skull. This superficial location, together with the small size of the brain, allows sampling of most or all of the IC through a small opening in the skull and dura. The preparation is mechanically very stable, with little movement caused by pulsation. To keep the surface of the brain moist and free of debris, the opening in the skull was covered with gel foam soaked in physiological saline solution at all times unless an electrode was inserted. Glass electrodes were double-pulled on a BrownFlaming P-87 horizontal electrode puller. The tips of the electrodes were $\sim 1 \mu \mathrm{m}$ in diameter and had resistances of 7-10 $\mathrm{M} \Omega$ when filled with internal solution. The internal solution contained (in $\mathrm{mM}$ ): 110 cesium gluconate, 11 EGTA, $10 \mathrm{CsCl}_{2}, 1 \mathrm{MgCl}_{2}, 1 \mathrm{CaCl}_{2}, 10 \mathrm{HEPES}, 2 \mathrm{ATP}$, and 0.3 GTP. The $\mathrm{pH}$ was adjusted to 7.2 , and the osmotic pressure to 280 mOsm. The formulation of the internal solution was optimized by trial and error. A cesium-based solution was chosen primarily because it was easier to form high-resistance seals using this solution than with solutions based on potassium. Cesium blocks some voltage-gated $\mathrm{K}^{+}$currents; dialysis of the intraccllular milicu climinates, or "washes out," synaptic responses attributable to G-protein-coupled receptors and may also reduce the contribution of some voltage-dependent ion channels. These characteristics have the advantage of permitting us to examine primarily ligand-gated currents and thus characterize fast synaptic inputs to the cell. A disadvantage is that we cannot fully characterize the natural course of spike generation, but this was not the primary aim of the study. The internal solution was prepared in advance, aliquoted, and stored for a maximum of 4 weeks at $-20^{\circ} \mathrm{C}$. In some experiments, $4 \%$ biocytin was added to the internal solution immediately before recording.

Recordings were made using an Axopatch 1-D patch-clamp amplifier. The head stage was attached to a custom-made clamp designed to fit on a Kopf hydraulic microdrive, so the electrode could be advanced by remote control from outside the sound-attenuating chamber. Pressure and suction were also applied to the electrode from outside the soundattenuating chamber via plastic tubing run through a port in the chamber wall. Unless otherwise stated, recordings were made in voltage-clamp mode. Data were digitized using a 16-bit analog-to-digital converter (A-D3, Tucker-Davis Technologies, Gainesville, FL) and collected using custom software developed in our laboratory.

The micropipette was positioned under visual control just above the surface of the IC. To prevent clogging, positive pressure was applied to the micropipette before lowering it into the tissue. The pressure was released after the tip reached an area from which recordings could be obtained. For many cells, it was possible to observe action potentials recorded extracellularly with the patch-clamp electrode before a seal was formed. These observations provided information about basic response properties of the cell, such as its best excitatory frequency and threshold, whether it was spontaneously active, and whether it was duration-tuned.

After a gigaohm seal was formed, breakthrough to whole-cell mode was achieved by applying gentle suction. In whole-cell mode, the resting membrane potential was measured and the cell was clamped at this resting potential during recording. Because of errors introduced by series resistance and dialysis of the cell by the cesium-containing internal solution, the precise membrane potential was not determined. Measured membrane potentials ranged from -30 to $-74 \mathrm{mV}$, with an average value of $-56.8 \pm 13 \mathrm{mV}(\mathrm{SD})$. Data were not collected from cells with measured membrane potentials more positive than $-30 \mathrm{mV}$, because it was assumed that they were damaged. All neurons from which recordings were obtained could be depolarized to threshold and thereby induced to fire action potentials. We were able to obtain stable whole-cell recordings from IC neurons for periods ranging from $10 \mathrm{~min}$ to $2.5 \mathrm{hr}$. In all of the neurons from which we recorded, both inward and outward currents were observed in response to sound. Voltage-clamp recordings were generally used to minimize the contribution of voltage-dependent currents and to more accurately measure the time course of synaptic currents. Because most neurons in the IC have extensive dendritic trees, voltage control was usually incomplete, allowing action potentials to escape clamp when the cell was depolarized by synaptic input. The outward currents we recorded are likely to be IPSCs attributable to activation of GABA or glycine receptors, and the inward currents are likely to be glutamatergic EPSCs. When using the terms "inhibition" or "excitation," we refer to the net effect of all the inputs, excitatory and inhibitory.

Auditory stimuli were generated using two digital signal processors each of which controlled a digital-to-analog converter (DA) (TuckerDavis Technologies), which were in turn controlled by custom software run on a Gateway 486 computer. The outputs of the D $\wedge$ s were routed through antialiasing filters to digitally controlled attenuators, amplified, and routed through a second set of attenuators to the loudspeakers at the two ears. Types of stimuli included pure tones, white noise, clicks, frequency modulated sweeps, sinusoidally amplitude-modulated signals (SAMs), and sinusoidally frequency-modulated signals (SFMs). For most experiments, a rise-fall time of $0.5 \mathrm{msec}$ was used. Stimuli always started at the zero crossing point of the sine wave. Presentation rate was $\sim 1 / \mathrm{sec}$ or less. Sounds were presented via Brüel and Kjaer 1/4-inch condenser microphones, modified for use as loudspeakers, and placed within the cone of the bat's pinna, as close as possible to the external ear canal. The outputs of the loudspeakers were measured with a 1/8-inch Brüel and Kjaer microphone and found to be flat $\pm 5 \mathrm{~dB}$ between 20 and $100 \mathrm{kHz}$, the frequency range used in the experiments. Using measurements taken in $1 \mathrm{kHz}$ steps, attenuator settings were converted to sound pressure level (SPL; re20 $\mu \mathrm{Pa}$ ).

After the final recording session, animals were administered a lethal dose of Nembutal (pentobarbital) and perfused through the heart with PBS followed by a fixation solution containing $4 \%$ paraformaldehyde in PBS. After perfusion, the brain was removed and stored overnight in $30 \%$ sucrose in PBS. Sections were cut $40 \mu \mathrm{m}$ thick on a freezing microtome. To visualize cells labeled with biocytin, sections were reacted with avidinbiotin complex (Vector Laboratories, Burlingame, CA) (Hsu et al., 1981).

\section{RESULTS}

The data reported here are based on recordings from 20 IC neurons in 13 animals. All of these neurons were in the dorsal half of the IC. In Eptesicus, the IC measures $\sim 2 \mathrm{~mm}$ in its entire dorsal-to-ventral extent. The depths of recordings ranged from 81 to $934 \mu \mathrm{m}$, with all but four between 100 and $300 \mu \mathrm{m}$. Many (13 of 20) of the neurons had at least occasional spontaneous EPSCs that caused breakaway action potentials to occur. Fewer neurons (3 of 20) had obvious spontaneous IPSCs. Three neurons had a very high rate of spontaneous spiking activity, and six showed no evidence of any spontaneous excitatory or inhibitory activity. These results are consistent with extracellular recordings in the IC that show that some spontaneous activity is present in the majority of neurons, but most neurons have low levels of spontaneous activity.

\section{Stimulus-evoked contralateral excitation and inhibition}

All but two neurons ( 18 of 20 , or $90 \%$ ) received contralateral excitatory input as seen in EPSCs evoked by sound presented to the contralateral ear. Often, these EPSCs were rapid and large enough to cause the neuron to reach spike threshold. This finding is qualitatively similar to all of the published extracellular recording data showing that the majority of IC neurons are excited by sound at the contralateral ear.

For 15 neurons, sufficient data were obtained to analyze the sequence of excitation and inhibition in response to sound at the 


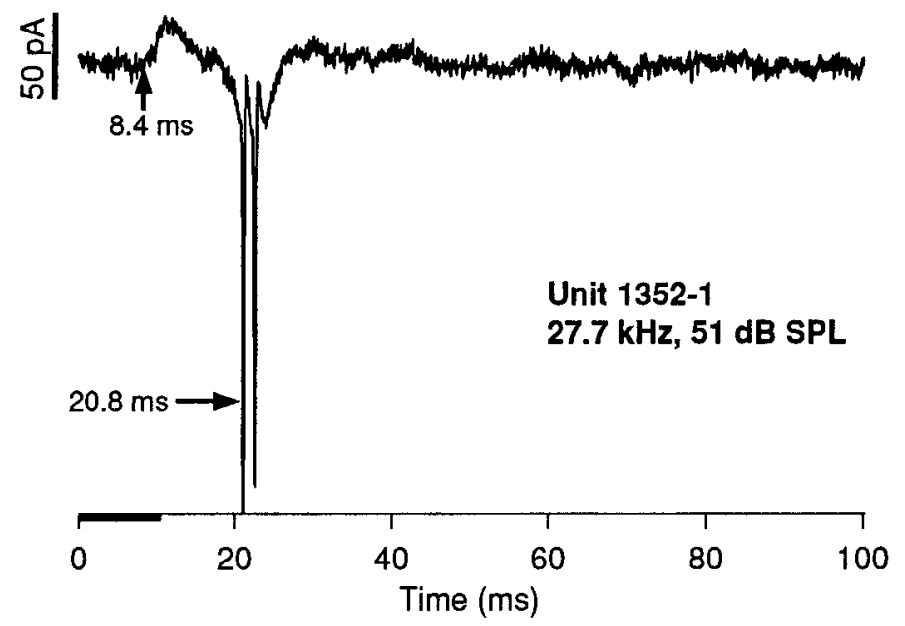

Figure 1. Response of an IC neuron to a $10 \mathrm{msec}$ pure-tone burst, showing a short-latency IPSC followed by an EPSC. Recording was in voltagc-clamp mode, with the cell held at its resting membrane potential of $-65 \mathrm{mV}$. On this and subsequent figures, the vertical scale bar indicates current in picoamperes $(p A)$ and the thick bar on the horizontal axis indicates the duration of the stimulus. In this and subsequent figures, upward deflections represent outward current or IPSCs. Downward deflections represent inward current or EPSCs.

contralateral ear under multiple stimulus conditions. For 13 $(87 \%)$, some stimulus conditions at the contralateral ear elicited IPSCs. As shown below, the time of occurrence of the IPSC was variable with respect to the EPSC.

\section{Leading inhibition}

Figure 1 illustrates an example of the response of a neuron with a short-latency IPSC followed by an EPSC. For this neuron, the IPSC began $\sim 8 \mathrm{msec}$ after the onset of a tone burst, reached a peak at $\sim 11 \mathrm{msec}$, and was followed by an EPSC and spikes at $\sim 20 \mathrm{msec}$ after stimulus onset. A similar pattern of an early IPSC followed by an EPSC was seen in 6 of 15 neurons under at least some stimulus conditions. We call this type of inhibition "leading inhibition" to distinguish it from "lagging inhibition," which followed excitation.

Assuming that leading inhibition is caused by release of GABA or glycine and acts via chloride channels, it should not have been affected by the presence of cesium in the electrode. Moreover, the finding of short-latency inhibition in IC neurons is consistent with extracellular recording studies that show that some IC neurons discharge earlier in response to sound when GABA or glycine receptor antagonists are applicd (Johnson, 1993; Park and Pollak, 1993b).

In some cases, leading inhibition was clearly responsible for lengthening the latency of the EPSC. Figure 2 shows an example of a neuron in which leading inhibition grew as the sound level of the stimulus increased. This neuron exhibited an increase in first-spike latency with increasing SPL, a phenomenon known as "paradoxical latency shift" (Sullivan, 1982). Paradoxical latency shift is sometimes seen in extracellular recordings from neurons at cortical (Sullivan, 1982) and subcortical (Covey, 1993) levels of the auditory system. The term "paradoxical" refers to the fact that the relationship between sound level and latency is the opposite of that seen in auditory nerve fibers and in most central auditory neurons. At $45 \mathrm{~dB}$ SPL, $10 \mathrm{~dB}$ above threshold, this neuron showed no evidence of an initial IPSC and fired action potentials with a latency of $15 \mathrm{msec}$. As sound level was increased to $65 \mathrm{~dB}$

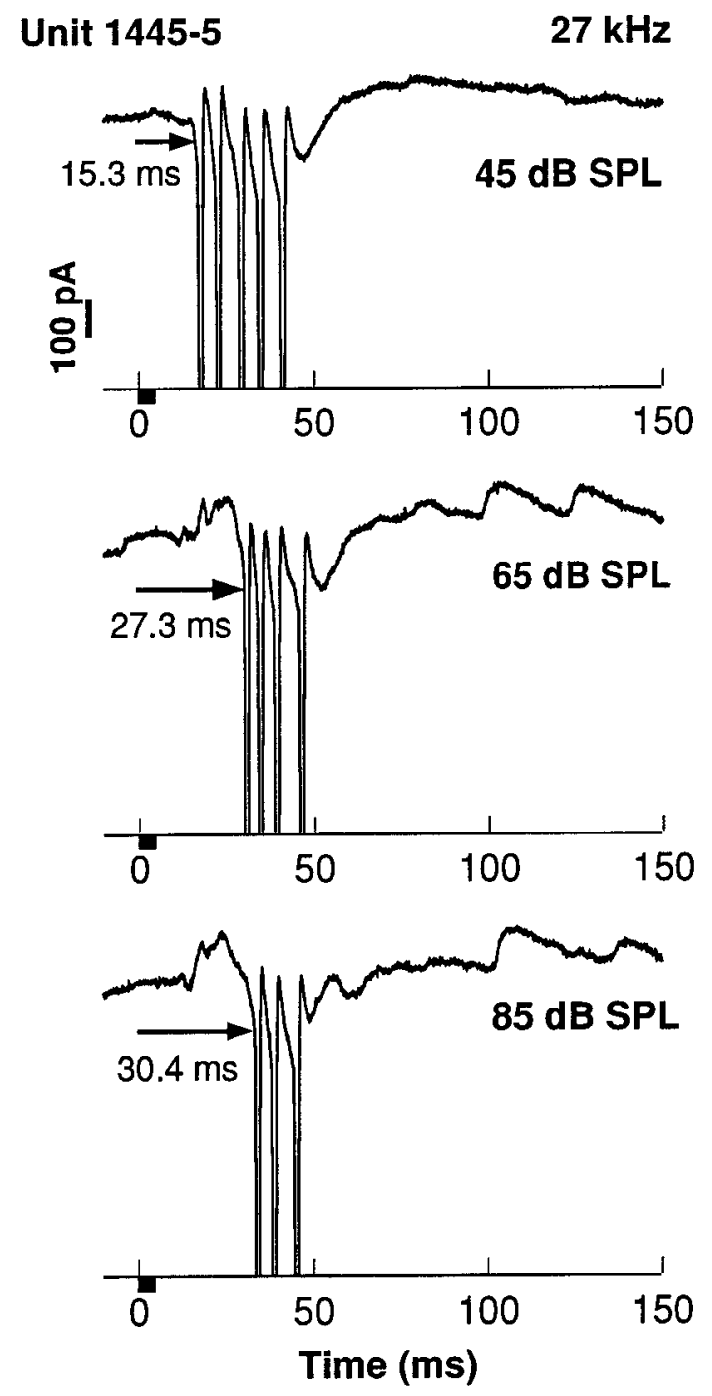

Figure 2. Growth of inhibition and increase in spike latency in response to $5 \mathrm{msec}$ pure-tone stimuli as SPL was increased. The SPL was increased from $45 \mathrm{~dB}(10 \mathrm{~dB}$ above threshold, top trace $)$ to $85 \mathrm{~dB}$. First-spike latency is indicated by the arrow on each trace. The resting potential of this ncuron was $-70 \mathrm{mV}$. Spikes have been truncated.

SPL, an IPSC appeared to cancel most of the initial portion of the excitation, eliminating the first spikes, so that response latency lengthened to $27 \mathrm{msec}$. Further increases in SPL resulted in a lengthening of the duration of the IPSC so that by $85 \mathrm{~dB}$ SPL, the response latency had lengthened to $30 \mathrm{msec}$. Note that sound level had little effect on the timing of the last spike.

\section{Lagging inhibition}

Figure 3 shows an example of a neuron that responded to a 20 msec pure tone with an outward current that followed the EPSC and spikes. Similar outward currents that followed an EPSC under at least some stimulus conditions were seen in 12 of 15 neurons $(80 \%)$. As in the case of leading inhibition, if the IPSC that followed the EPSC was attributable to GABAergic or glycinergic synaptic input, it should not have been affected by the presence of cesium in the electrode. The duration of the lagging inhibition ranged from $\sim 5 \mathrm{msec}$ to at least $150 \mathrm{msec}$, the longest recording period used. In five neurons, the EPSC was flanked by both early and late inhibition as in Figure 3; in seven neurons, only lagging inhibition was seen. Extracellular recordings have shown that 


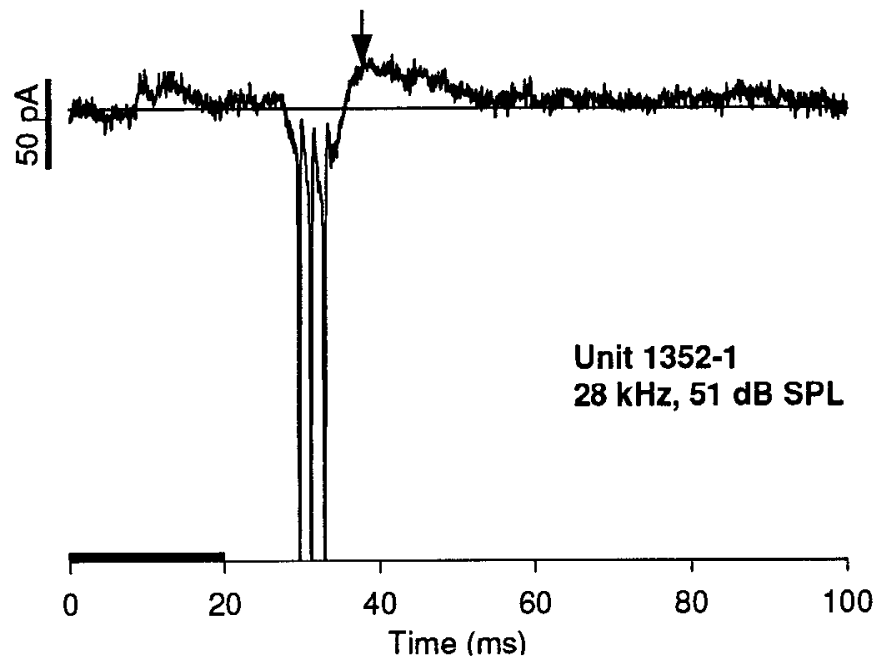

Figure 3. The same neuron illustrated in Figure 1 responded to a $20 \mathrm{msec}$ pure tone with a small, short-latency IPSC (latency $=8.2 \mathrm{msec}$ ) followed by an EPSC correlated with sound offset (latency to spike $=29.4 \mathrm{msec}$ ). The EPSC was followed by a second IPSC (arrow) that lasted for $\sim 20$ msec. To better illustrate the late IPSC, a thin line is drawn through the baseline. Spikes have been truncated.

applying antagonists of GABA or glycine receptors causes transient onset responses to lengthen (Faingold et al., 1991; Johnson, 1993; Pollak and Park, 1993) and suggests that the lagging inhibition seen in our recordings was attributable to synaptic input.

For neurons like the one shown in Figure 3, the fact that there is outward current both before and after the EPSC raises the question of whether early and late inhibition are caused by separate inputs or whether they represent the beginning and end of a single, long-lasting IPSC triggered by stimulus onset and interrupted by a large, short-duration EPSC. Although $\mathrm{K}^{+}$currents were theoretically blocked in our experiments because of the presence of cesium in the electrode, we cannot rule out the possibility that some intrinsic conductances remained active. Thus, the late outward current might have been afterhyperpolarization after the synaptically mediated EPSC. To resolve this issue, it will be necessary in future experiments to use methods to block different specific intrinsic conductances and to look at differences between spontaneous and evoked spikes.

\section{Inhibitory effects in the absence of synaptic inhibition}

Figure 4 illustrates that it is possible to distinguish between inhibition occurring at IC neurons and inhibition that has occurred elsewhere. The neuron in Figure 4 had a very high rate of spontaneous EPSCs and action potentials that could be observed in extracellular recording before seal formation, as well as intracellularly (top trace). This spontaneous activity was completely eliminated by presentation of a sound at the contralateral ear. Because there was little evidence of synaptic inhibition in either voltage-clamp mode (middle trace) or current-clamp mode (bottom trace), it seems likely that this neuron received excitatory input from a second, tonically active neuron that was inhibited by sound at the contralateral ear. It is very unlikely that the high rate of spontaneous activity could have been attributable to injury because we were able to obtain stable and reliable recordings for $>1 \mathrm{hr}$. One other neuron had a similar high level of spontaneous activity but did not show any obvious response to any of the auditory stimuli tested. Even in extracellular recording experiments, IC neurons with very high spontaneous activity are some-
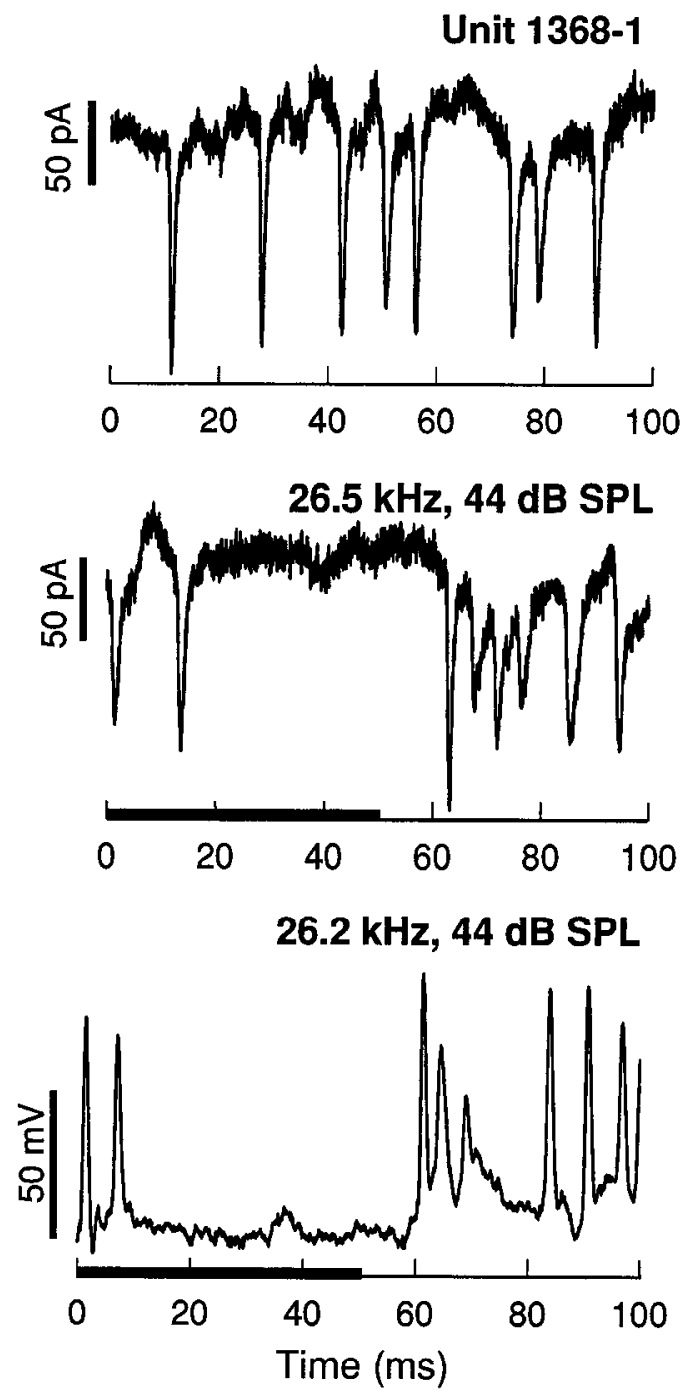

Figure 4. Inhibition of spiking in a neuron with a high rate of spontaneous activity. The top trace shows spontaneous activity in the absence of a stimulus; the middle and bottom traces show the neuron's response to 50 msec pure tones. The rriddle trace was recorded in voltage-clamp mode, the bottom trace in current-clamp mode. The resting potential of this neuron was $-65 \mathrm{mV}$.

times encountered, especially in the dorsal part of the IC, where most of our patch-clamp recordings were obtained.

\section{Poststimulus oscillations}

Figure 5 shows the response of a neuron in which prolonged oscillations followed the response to a $20 \mathrm{msec}$ pure tone and persisted for at least $100 \mathrm{msec}$ after the end of the EPSC. For this neuron, the oscillations occurred at a rate of $\sim 30 \mathrm{~Hz}$. The oscillations were present across a wide range of stimulus amplitudes starting at $10 \mathrm{~dB}$ above threshold, and across a wide range of frequencies. Of the neurons from which we recorded, nearly half (7 of 15) had oscillatory currents that persisted for a prolonged time period after the excitatory component of the response. The observation of oscillatory currents after responses to sound is consistent with extracellular studies showing that some IC neurons undergo periodic waxing and waning of responsiveness to the second of a pair of tones separated by a variable interval (Grinnell, 1963; Suga, 1964).

Although the oscillations appear to be caused by periodic 


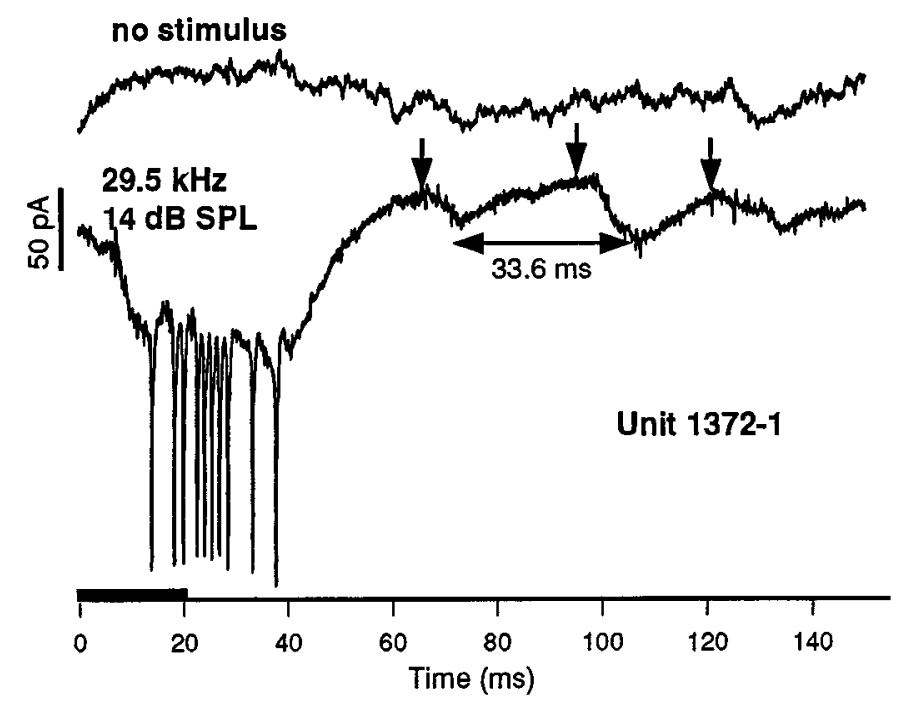

Figure 5. Response of an IC neuron that exhibited oscillations after the excitatory response evoked by a $20 \mathrm{msec}$ pure tone. The top trace was recorded in the absence of a stimulus. The bottom trace shows the neuron's response to a tone. The oscillations (arrows) occurred at a rate of $\sim 30 \mathrm{~Hz}$ and persisted for at least $100 \mathrm{msec}$. The resting potential of this neuron was $-64 \mathrm{mV}$

outward currents, they could represent either delayed synaptic inputs or intrinsic oscillatory properties of the cell triggered by the synaptically mediated EPSC. Further experiments will be necessary to determine the origin of the oscillations, including depolarizing the cell to determine whether oscillations follow spikes that are not synaptically mediated.

\section{Frequency tuning: inhibitory side bands}

For all of the neurons from which we recorded, the sequence and time course of inhibitory and excitatory synaptic events varied considerably as a function of stimulus parameters such as SPL, duration, and frequency. For eight neurons, frequency was varied systematically so that responses were recorded not only within the excitatory portion of the tuning curve, but also at frequencies above and below. All of these neurons exhibited EPSCs evoked by sound over some range of frequencies. Six neurons responded with clear sound-evoked IPSCs at frequencies above and below the bandwidth that produced an EPSC. Two neurons did not show any evidence of sound-evoked IPSCs at frequencies above or below the excitatory region. Figure 6 illustrates the responses of a neuron the best frequency of which, as defined by spike output, was $26 \mathrm{kHz}$. At this frequency, the neuron responded with an EPSC accompanied by action potentials (lower trace). Decreasing the frequency by $1 \mathrm{kHz}$, to $25 \mathrm{kHz}$ (top trace), the response changed from robust excitation to an IPSC with a latency slightly longer than that of the EPSC evoked by $26 \mathrm{kHz}$. At frequencies higher than the neuron's excitatory range, an IPSC also appcarcd (middle trace). The latency of the IPSC evoked by $29 \mathrm{kHz}$ was longer than that of the IPSC evoked by $25 \mathrm{kHz}$. At frequencies both above and below the excitatory range, no initial inward current was present, suggesting that the excitatory input to the neuron was inactive at these frequencies, not simply canceled by the IPSC. In 6 of 10 trials, a spike followed the IPSC, either from delayed excitatory input at this frequency or rebound from inhibition.

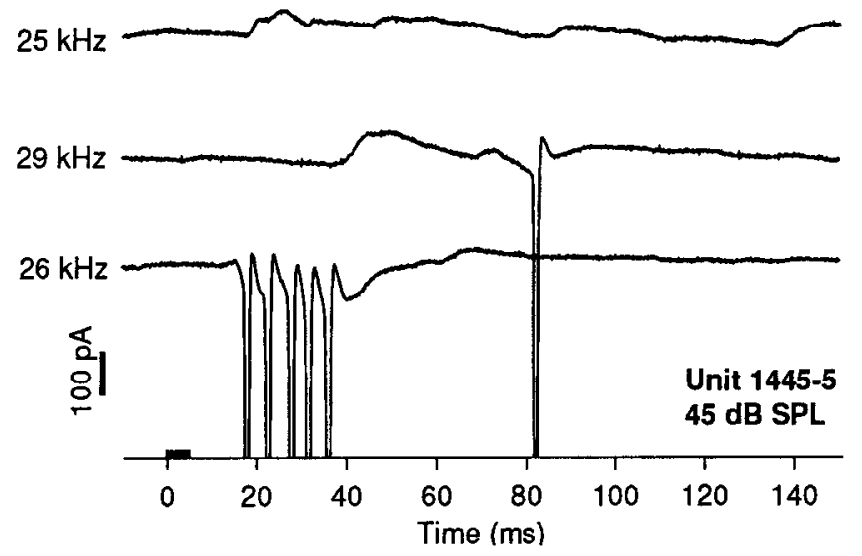

Figure 6. Responses of an IC neuron to a $5 \mathrm{msec}$ pure tone at different frequencies. Note that frequencies are arranged in nonsequential order. The response to a tone at the neuron's best frequency $(26 \mathrm{kHz}$, bottom trace) was almost exclusively excitatory. A tone above best frequency ( 29 $\mathrm{kHz}$, middle trace) elicited a long-latency IPSC. A tone below best frequency $(25 \mathrm{kHz}$, top trace) elicited a short-latency IPSC. This neuron's resting potential was $-70 \mathrm{mV}$. Spikes have been truncated.

\section{A delicate balance of inhibition and excitation is revealed by changes in sound level}

For some neurons, it was possible to determine that the amplitude of sound influenced the relative proportion of synaptic inhibition and excitation. In extracellular studies of the IC, it is common to find neurons with an upper spike threshold for sound level so that they fire only when sound level is within an intermediate range (Casseday and Covey, 1992). This means that they have closed frequency tuning curves. Eight neurons with closed tuning curves were tested over a wide range of sound amplitudes that began below the lower excitatory threshold and extended above the upper excitatory threshold.

Figure 7 shows an example of such a neuron that responded with an IPSC at high sound levels above its upper spike threshold, but not below. For this neuron, at $24 \mathrm{~dB}$ SPL, there was essentially no synaptic response. At $34-44 \mathrm{~dB}$ SPL, there was a small

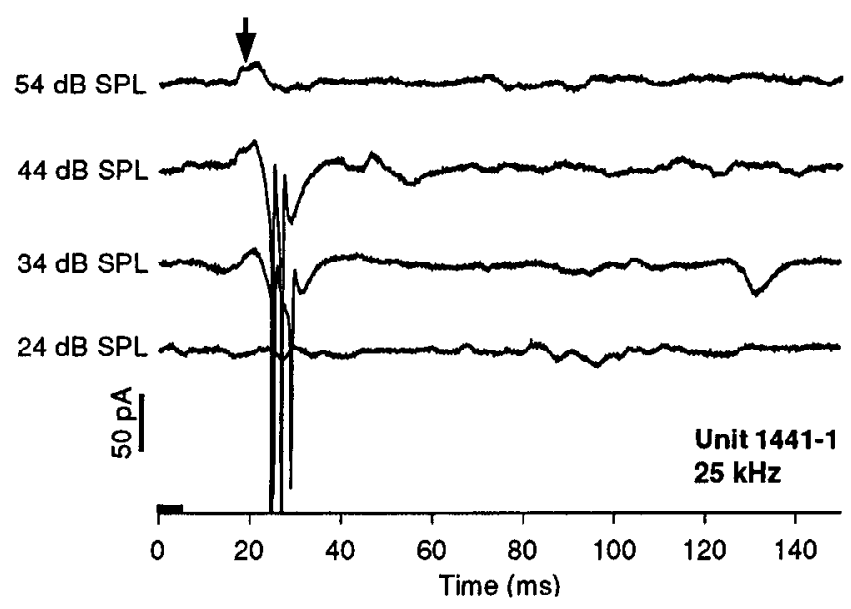

Figure 7. Responses of an IC neuron to a $5 \mathrm{msec}$ pure tone at different sound levels. The traces are arranged with low sound levels at the bottom and high sound levels at the top. There are no spikes in the top or bottom trace. The arrow points to the IPSC that persisted at high sound levels above the upper excitatory threshold of the neuron. This neuron had a resting potential of $-59 \mathrm{mV}$. Spikes in the trace at $34 \mathrm{~dB}$ SPL have been truncated. 


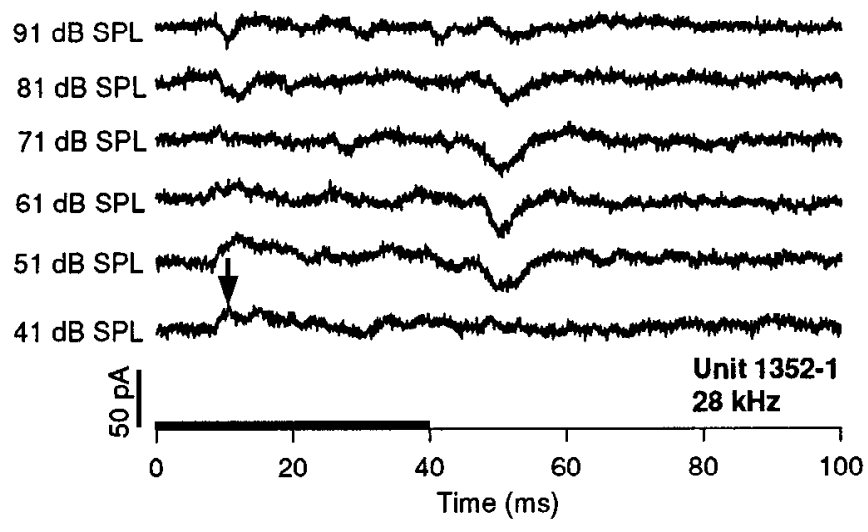

Figure 8. Responses of the same IC neuron illustrated in Figure 1 to a 40 msec pure tone at different sound levels. The traces are arranged with low levels at the bottom and high levels at the top. The arrow points to the IPSC that was present at low sound levels.

short-latency IPSC followed immediately by an EPSC and spikes. At $54 \mathrm{~dB}$ SPL, the EPSC had almost entirely disappeared, but the IPSC persisted, and no spike was evoked at this or higher sound levels. The predominance of the IPSC at high sound levels presumably is responsible for the limited dynamic range of the spike output in cells with closed tuning curves.

Figure 8 shows the responses of a duration-tuned neuron tested at a long sound duration that did not elicit spikes. In this neuron, the balance of inhibition and excitation was reversed from that in the previous example. For this neuron, the inhibitory threshold was lower than the excitatory threshold. At $41 \mathrm{~dB}$ SPL, the only response was a small IPSC with a latency of $8.6 \mathrm{msec}$. At $51 \mathrm{~dB}$ SPL, the initial IPSC was larger, and a late inward current appeared, correlated in time with sound offset. By $71 \mathrm{~dB}$ SPL, the initial IPSC had disappeared, and at 81 and $91 \mathrm{~dB}$ SPL it was replaced by a short-latency FPSC. Note that although this cell received both excitatory and inhibitory inputs over a dynamic range of $50 \mathrm{~dB}$, the balance of excitation to inhibition offset one another, so that the neuron never produced a spike.

\section{Duration tuning}

Extracellular recordings show that approximately one-third of all IC neurons in Eptesicus are tuned to specific sound durations (Casseday et al., 1994). Figure 9 shows the responses of a duration-tuned neuron to pure tones of different durations. Extracellular recordings before seal formation showed that this neuron was duration-tuned with a best duration of $\sim 10 \mathrm{msec}$. This neuron responded to all sounds with an initial short-latency IPSC that began at 8-9 msec after sound onset. An EPSC occurred later with a latency that was clearly correlated with the offset of the sound. For a $5 \mathrm{msec}$ stimulus, the EPSC immediately followed the initial IPSC, but there were seldom any spikes. For a $10 \mathrm{msec}$ stimulus, the EPSC latency was longer, and the neuron fired multiple spikes. For a 20 msec stimulus, the latency of the EPSC was still greater, and the neuron only occasionally fired 1-2 spikes. As stimulus duration was increased to 30 and $40 \mathrm{msec}$, the EPSC latency became progressively longer and its magnitude smaller. Spikes were rarely fired in response to these long-duration stimuli (see Fig. 8). One interpretation of this behavior is that the duration tuning of this neuron was attributable to three stimuluslocked events: (1) an initial inhibitory input that lasts as long as the sound, seen as the initial IPSC; (2) a transient excitatory input, the latency of which is correlated with stimulus onset, but which
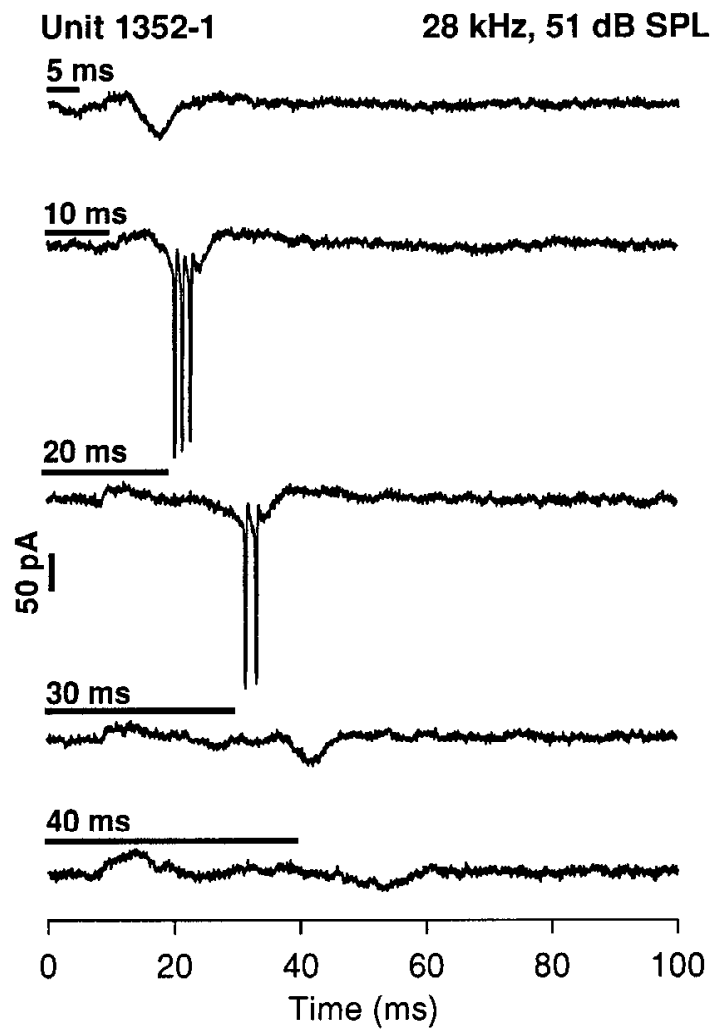

Figure 9. Responses of the same IC neuron shown in Figure 1 to pure tones of different durations. The traces are arranged with short durations at the top and long durations at the bottom. The bar above each trace indicates the sound duration.

arrives later than the initial inhibitory input and cither counterbalances it or results in a small net inward current; and (3) an offset excitation, seen as inward current correlated with the time of sound offset. Because the onset EPSC is counterbalanced by inhibition, it alone is never sufficient to cause the neuron to fire. The offset excitation alone is also subthreshold. Only when sound duration is such that the onset excitation coincides with the offset excitation does the neuron reach its spike threshold and fire. The validity of this or other models could be tested in future experiments through manipulation of the reversal potential for the IPSC.

\section{Responses to modulated stimuli}

Most neurons in the IC respond only to the onset of sound. We tested one such neuron with SAM. Figure 10 shows the responses of this neuron to SAM stimuli at different modulation rates. The neuron had a low-pass characteristic, responding with an onset discharge to modulation frequencies of $\leq 120 \mathrm{~Hz}$. After the onset response, there was a cyclic pattern of synaptic currents that persisted throughout the entire duration of the stimulus and followed the modulation cycles of the signal. The cyclical modulations were most prominent at 30 and $60 \mathrm{~Hz}$. At $100 \mathrm{~Hz}$, the synchrony became degraded over the course of the response. The cyclic response pattern appeared to be attributable to periodic inward currents, the form and time course of which approximated the amplitude envelope of the stimulus, interspersed with periodic returns to baseline. This cyclic activity suggests that the neuron received excitatory inputs from neurons that responded to the amplitude-modulated stimulus in a phase-locked manner.

A similar cyclic pattern was seen in the responses of this neuron 


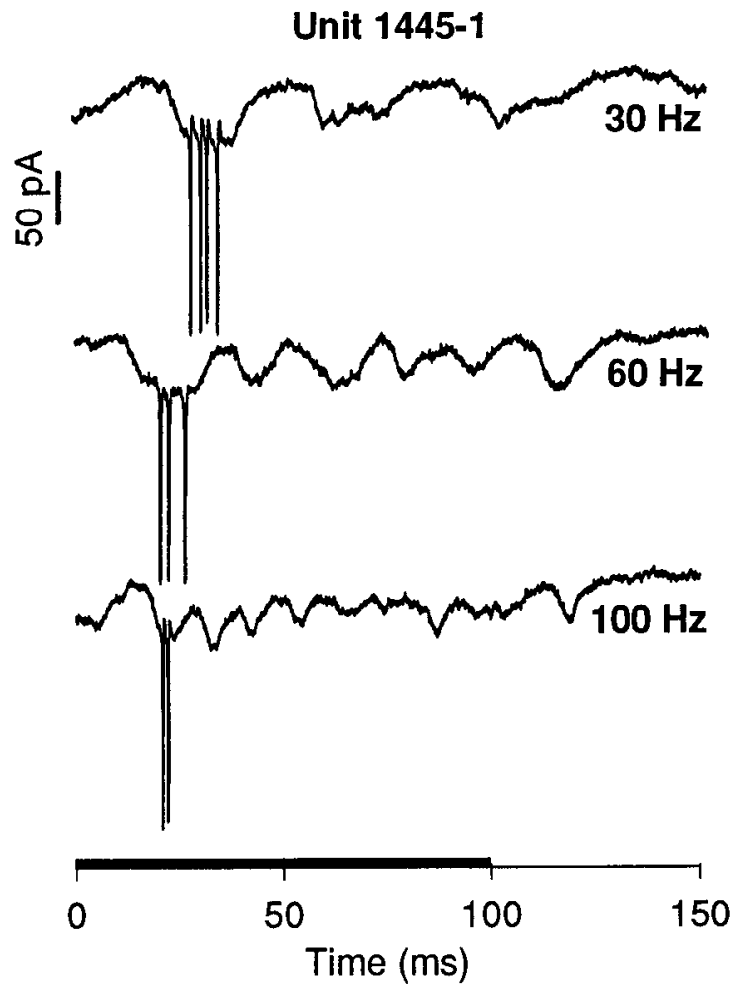

Figure 10. Responses of an IC neuron to $100 \mathrm{msec}$ SAM signals modulated at three different rates. The carrier frequency was $26 \mathrm{kHz}$, and the sound level was $35 \mathrm{~dB}$ SPL. The modulation rates are indicated at the right of each trace. The resting potential of the neuron was $-67 \mathrm{mV}$.

to an SFM stimulus and in the responses of one other neuron that we tested with SAM and SFM stimuli. Neither of these ncurons appeared to be specialized for modulated stimuli because they also responded to pure tones.

The pattern of synaptic currents seen in response to the SAM signal suggested that this neuron received an input signal, the magnitude of which was proportional to the amplitude envelope of the stimulus. To further test this idea, we varied the rise time of a $100 \mathrm{msec}$ pure tone. The neuron's responses to variations in the rise time are shown in Figure 11. As would be predicted if the response were proportional to the amplitude of the envelope, the longer the rise time the more gradual was the slope of the EPSC leading to the point at which the neuron spiked. After the burst of spikes, a small EPSC gradually decayed throughout the remainder of the stimulus duration.

\section{Binaural interactions}

For two neurons, we were able to obtain series of recordings that showed clear evidence of binaural interaction based on EPSCs evoked by contralateral stimuli and IPSCs evoked by ipsilateral stimuli.

Both neurons responded in a very similar way to binaural stimulation. Figure 12 shows the responses of one neuron to various combinations of ipsilateral and contralateral sound. The contralateral stimulus alone at a sound level of $45 \mathrm{~dB}$ SPL elicited a large EPSC and a burst of spikes (Fig. 12A). Evidence of binaural inhibition first became apparent when the ipsilateral level was set at $10 \mathrm{~dB}$ above that of the contralateral stimulus (Fig. $12 B$ ). This binaural combination partially canceled the later part of the EPSC. When the contralateral stimulus was turned off but the ipsilateral stimulus remained at the same level (Fig. 12C),

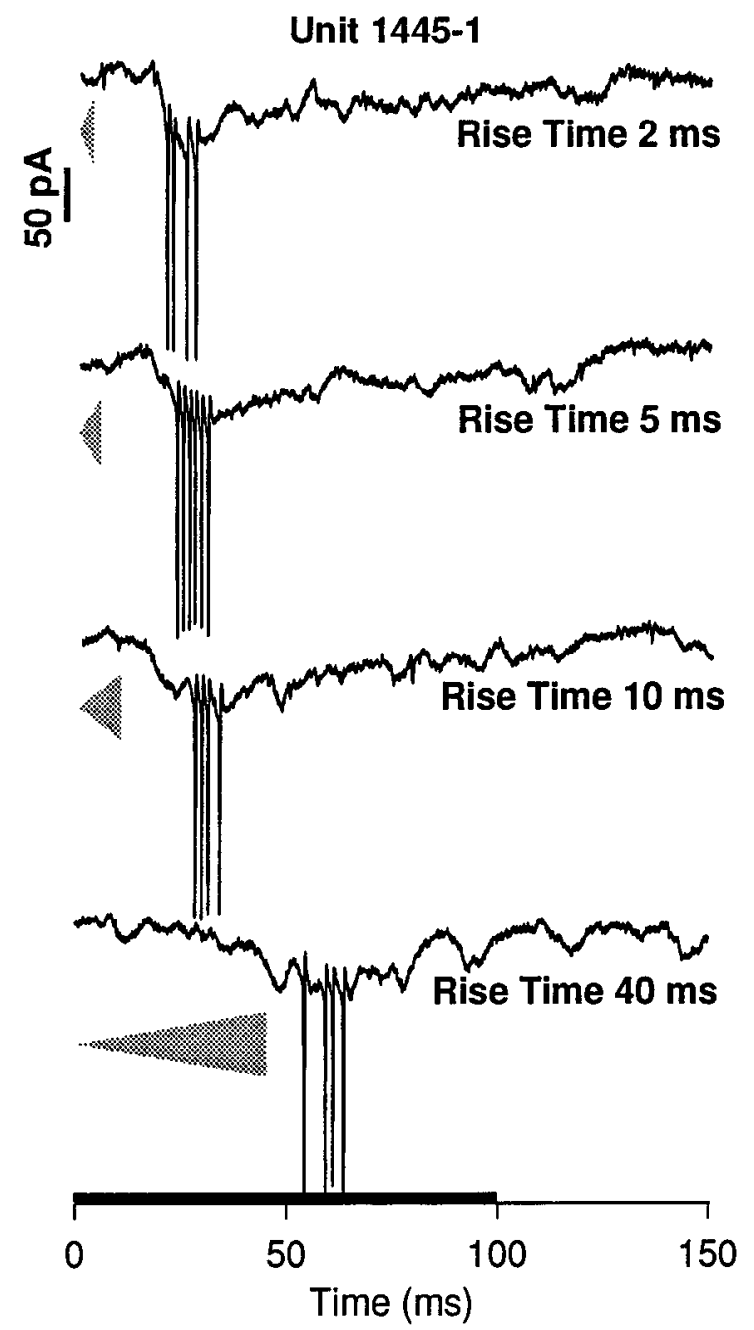

Figure 11. Responses of the same neuron illustrated in Figure 10 to changes in the rise time of a $100 \mathrm{msec}$ pure tone at $26 \mathrm{kHz}$ and $35 \mathrm{~dB}$ SPL. Shaded symbols indicate the rise time over which sound amplitude was increased.

there was a small, rather variable IPSC that was sometimes followed by a spike. When the level of the ipsilateral stimulus was increased to $20 \mathrm{~dB}$ above that of the contralateral one (Fig. 12D), the EPSC and spikes were completely eliminated, and only a long-lasting IPSC was present. The ipsilateral sound alone at this level (Fig. 12E) elicited a large sustained IPSC, sometimes followed by a spike. The long time course of these responses is significant because the duration of this IPSC was longer than that of the contralaterally evoked EPSC. When a spike occurred after the IPSC, it was $>50 \mathrm{msec}$ after stimulus onset. In addition, the size of the ipsilaterally evoked IPSCs in the neurons in which they were observed was typically larger than that of any of the contralaterally evoked IPSCs in other neurons. A similar pattern of contralateral excitation and ipsilateral inhibition, including the long-latency spike after ipsilateral sound, was observed in one other cell. The long-latency spike never occurred in response to the contralateral sound, suggesting that there may be some lagging inhibition in the contralateral input. If these temporal properties of excitation and inhibition are typical of binaural cells in the IC, then the time frame over which these cells integrate binaural information must be much longer than previously realized. 

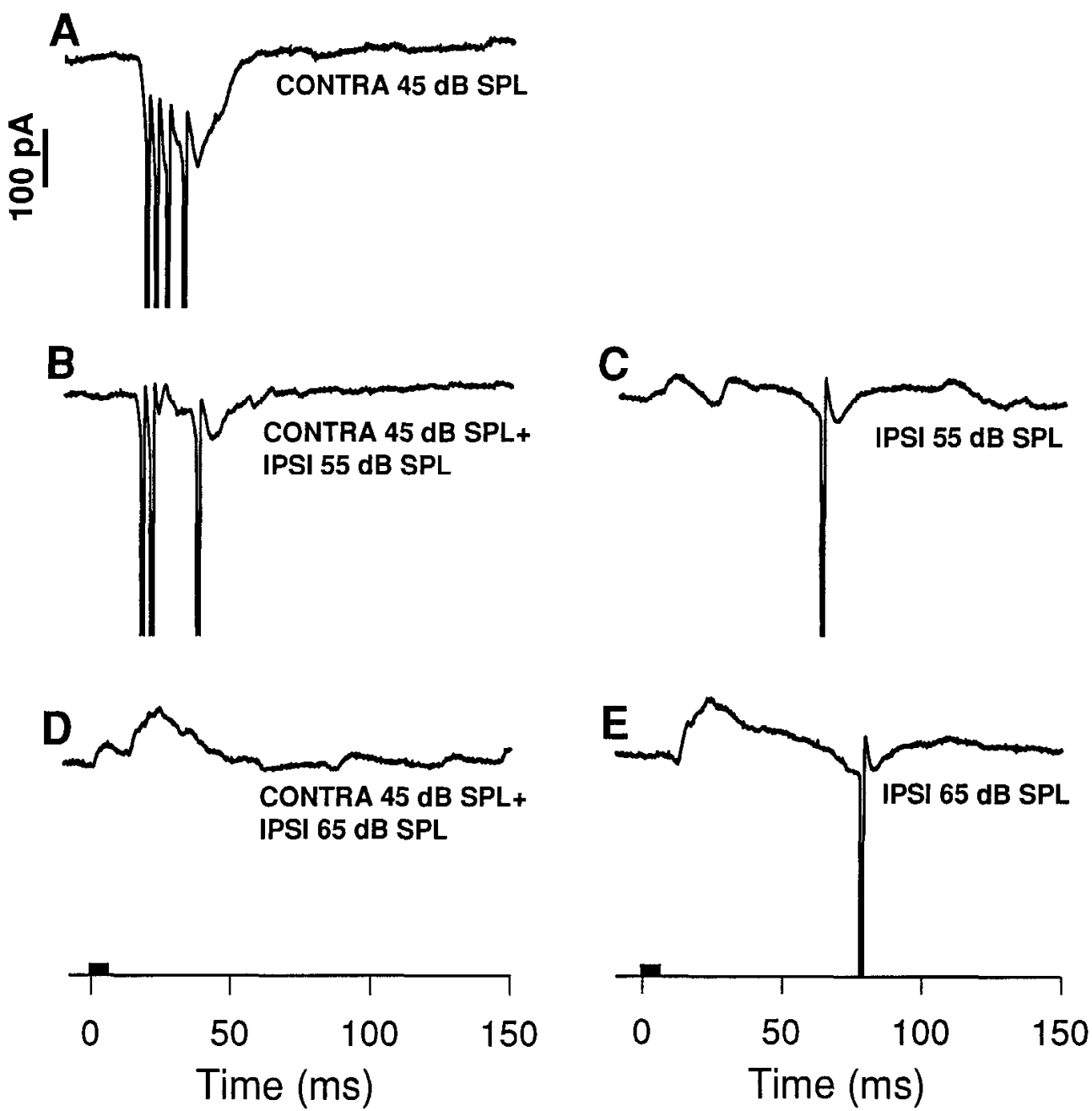

Figure 12. Binaural interaction in an IC neuron that responded to contralateral sounds with an EPSC and ipsilateral sounds with an IPSC. In the traces in the left column, the contralateral sound is a $5 \mathrm{msec}$ pure tone at $27 \mathrm{kHz}$ and $45 \mathrm{~dB}$ SPL, either alone (top trace) or in combination with a simultaneous ipsilateral tone of the same frequency at the sound levels indicated. The traces on the right show the responses to ipsilateral tones alone. The resting potential of this neuron was $-70 \mathrm{mV}$. Spikes in all traces have been truncated.

\section{DISCUSSION}

We began this study as a search for an efficient method to test the idea that the different types of highly specialized processing found in the IC are created through convergence of excitatory and inhibitory synaptic inputs. Although only a few types of such specialized processing are described here, in the following discussion we show how this method can answer fundamental questions concerning the integration of sensory information.

\section{Temporal interplay of monaural excitation and inhibition}

Some of the most interesting questions concerning integrative processes in the CNS have to do with the mechanisms for processing time-varying information. In audition, time-varying parameters can be as simple as the duration of a pure tone or as complex as the pattern of spectral-temporal changes in speech or music. One fundamental mechanism that may underlie the processing of time-varying information is the convergence of inputs from neural delay lines. These inputs may be inhibitory or excitatory, and they may be transient or sustained (Casseday and Covey, 1996b). Our results directly reveal mechanisms for creating delays, modifying discharge patterns, and establishing tuning for specific features of sound such as duration.

\section{Leading inhibition}

For approximately one-third of the neurons in our study, the earliest response to sound was an IPSC. This finding provides direct evidence that IC neurons receive short-latency inhibitory input that lengthens response latency. In the IC, the range of latencies is considerably larger than would be expected from synaptic delays and axon length (Haplea et al., 1994; Casseday and Covey, 1996b). First-spike latency of IC neurons decreases when either GABAergic or glycinergic inhibition is blocked (Johnson, 1993; Park and Pollak, 1993b; Casseday and Covey, 1996a). Intracellular recording in anesthetized cats has shown sound-evoked IPSPs preceding EPSPs (Nelson and Erulkar, 1963).

These results demonstrate an important new principle. The balance between excitation and inhibition can vary systematically as a function of stimulus parameters. For example, in a neuron that exhibited "paradoxical latency shift" (Sullivan, 1982), the magnitude and duration of leading inhibition increased as a function of sound level, causing response latency to increase.

\section{Lagging inhibition}

A long-lasting outward current commonly followed sound-evoked spikes. The magnitude and time course of this current varied as a 
function of parametric changes in stimulus conditions. These results may explain the finding that the majority of IC neurons respond transiently to sound, even though the majority of auditory neurons at lower levels respond with a sustained discharge (Rose et al., 1963). Nelson and Erulkar (1963) showed that in the IC of the anesthetized cat, long-lasting hyperpolarization followed spikes evoked by clicks, but did not follow spontaneous spikes. Kuwada et al. (1980) showed intracellular recordings from one IC. neuron that responded to sound with a transient burst of action potentials followed by a period of hyperpolarization $\sim 100 \mathrm{msec}$ in duration. Local blocking of GABA or glycine causes some transient onset responses to lengthen, indicating that excitatory input is sustained (Faingold et al., 1991; Johnson, 1993; Pollak and Park, 1993).

Many IC neurons cannot follow repetition rates above a few hundred Hertz, whereas the responses of most neurons at lower levels to modulated stimuli are constrained only by their refractory periods. Although they may not fire on every cycle, these neurons continue to phase-lock at rates up to several thousand Hertz (Grinnell, 1963; Suga, 1964; Casseday and Covey, 1996a). In our study, the long periods of postexcitatory inhibition, $5 \mathrm{msec}$ to $>150 \mathrm{msec}$, provide an explanation for this limitation in response to high repetition rates.

\section{Oscillations}

Oscillatory currents that follow an EPSC may be the mechanism that underlies the observation that IC neurons respond only over a low and limited range of modulation rates. They may also underlie the observation that the response to the second sound of a pair may alternate between periods of recovery or enhancement and periods of suppression (Grinnell, 1963; Suga, 1964). Many aspects of sound-evoked oscillations remain to be understood, including the issue of whether they are synaptic or intrinsic in their origin.

\section{Duration tuning}

Approximately one-third of the neurons in the IC of Eptesicus respond only to a narrow range of sound durations and do not respond to shorter or longer durations. The responses of duration-tuned neurons are transient and their latency is always longer than their best duration. Local blocking of GABA or glycine abolishes duration tuning (Casseday et al., 1994). Wholecell recording on a typical duration-tuned cell showed that leading inhibition dominated the early part of the response and that there was an inward current associated with sound offset. These observations demonstrate the importance of the interaction of excitatory and inhibitory inputs offset in time from one another in creating tuning to simple temporal features of a stimulus (Casseday and Covey, 1996a).

\section{Spectral-temporal interactions}

Our results show that $\mathrm{IC}$ neurons receive synaptic inputs from sources tuned to different frequencies. Furthermore, these different inpuls may have different thresholds, different latencies, and different time courses. Many IC neurons have inhibitory areas surrounding their excitatory frequency areas (Casseday and Covey, 1992). Pharmacological studies of IC neurons have shown that blocking inhibition may cause the frequency response area to broaden or the threshold to decrease (Faingold et al., 1991; Vater et al., 1992; Yang et al., 1992). Although one important function of inhibition may be to shape the frequency tuning curve, our data suggest that another function may be to tune IC neurons to specific directions or rates of frequency change. The IC contains neurons that respond only to an upward or downward frequency sweep; some of these neurons only respond to a specific sweep duration (Grinnell, 1963; Suga, 1964; Casseday and Covey, 1992; Casseday et al., 1994; Fuzessery, 1994). The whole-cell method offers an experimental strategy for lesting different models for the creation of tuning to the direction of a frequency sweep (Fuzessery, 1994; Casseday and Covey, 1996a).

\section{Binaural interaction of excitation and inhibition}

Although the first stages of binaural processing clearly occur below the IC, neuropharmacological studies (Faingold et al., 1991; Park and Pollak, 1993a) and intracellular recording (Nelson and Erulkar, 1963) show that further binaural interactions take place at the cellular level in the IC. Characterizing the time course of ipsilateral inhibitory input relative to contralateral excitatory input is particularly important for evaluating ideas that have been proposed concerning the contribution of the IC to binaural processing. One idea is that the IC is the site of suppression to a second sound presented later and at a different spatial location from the first, the so-called "precedence effect" (Yin, 1994). A second hypothesis is that tuning for the dircetion of a moving sound source depends on prolonged ipsilateral inhibition (Moiseff, 1985). The fact that ipsilateral inhibition was long lasting in the cells from which we recorded suggests that it might indeed be involved in one or both of these processes.

An issue that has received little attention is whether IC neurons might receive a combination of ipsilateral and contralateral inhibition that shapes both binaural and monaural response properties. Our results indicate that at least some IC neurons do receive both ipsilateral and contralateral inhibition. For example, the neuron illustrated in Figures 6 and 12 received ipsilateral inhibition that suppressed the contralateral response as well as contralateral inhibition at frequencies above and below its best frequency. By directly observing synaptic events, it will be possible in future studies to determine how inhibition from ipsilateral and contralateral sources interact to shape complex binaural response propertics of IC ncurons.

\section{Conclusion}

Tuning for specific stimulus features or patterns can be created through convergence of excitatory and inhibitory delay lines with different discharge patterns. As a consequence of this convergence, a simple brief stimulus can evoke a long-lasting sequence of inhibitory and excitatory synaptic events in IC cells. This sequence of events can create temporal filters for simple features of sound such as duration or direction of frequency change, and probably for more complex patterns such rate of modulations in amplitude or frequency. It should now be possible with the wholecell method to measure the amplitude and duration of synaptic events that are evoked by sensory stimuli and to separate the effects of synaptic inputs from those of voltage-dependent conductances.

\section{REFERENCES}

Blanton MG, Lo Turco JJ, Kriegstein AR (1989) Whole cell recording from neurons in slices of reptilian and mammalian cerebral cortex. J Neurosci Methods 30:203-210.

Casseday JH, Covey E (1992) Frequency tuning properties of neurons in the inferior colliculus of an FM bat. J Comp Neurol 319:34-50.

Casseday JH, Ehrlich D, Covey E (1994) Neural tuning for sound duration: role of inhibitory mechanisms in the inferior colliculus. Science 264:847-850. 
Casseday JH, Covey $\mathrm{E}$ (1996a) Mechanisms for analysis of auditory temporal patterns in the brainstem of echolocating bats. In: Neural representation of temporal patterns (Covey E, Hawkins HL, Port RF, eds), pp 25-52. New York: Plenum.

Casseday JH, Covey E (1996b) A neuroethological theory of the operation of the inferior colliculus. Brain Behav Evol, in press.

Covey E (1993) Response properties of single units in the dorsal nucleus of the lateral lemniscus and paralemniscal zone of an echolocating bat. J Neurophysiol 69:842 859 .

Covey E, Casseday JH (1995) The lower brainstem auditory pathways. In: Springer handbook of auditory research, Vol 5, Hearing by bats (Popper AN, Fay RR, eds), pp 235-295. New York: Springer.

Edgar PP, Schwartz RD (1990) Localization and characterization of ${ }^{35} \mathrm{~S}$ t-butylbicyclophosphorothionate binding in rat brain: an autoradiographic study. J Neurosci 10:603-612.

Faingold CL, Boersma-Anderson CA, Caspary DM (1991) Involvement of GABA in acoustically-evoked inhibition in inferior colliculus neurons. Hear Res 52:201-216.

Fenwick EM, Marty A, Neher E (1982a) A patch-clamp study of bovine chromaffin cells and of their sensitivity to acetylcholine. I Physiol (Lond) 331:577-597.

Fenwick EM, Marty A, Neher E (1982b) Sodium and calcium channels in bovine chromaffin cells. J Physiol (Lond) 331:599 635.

Ferster D, Jagadeesh B (1992) EPSP-IPSP interactions in cat visual cortex studied with in vivo whole-cell recording. $\mathrm{J}$ Neurosci $12: 1262-1274$

Fubara BM, Casseday JH, Covey E, Schwartz-Bloom RD (1996) Distribution of $\mathrm{GABA}_{\mathrm{A}}, \mathrm{GABA}_{\mathrm{B}}$ and glycine receptors in the central auditory system of the big brown bat, Eptesicus fuscus. J Comp Neurol, in press.

Fuzessery ZM (1994) Response selectivity for multiple dimensions of frequency sweeps in the pallid bat inferior colliculus. J Neurophysiol 72:1061-1079.

Glendenning KK, Baker BB (1988) Neuroanatomical distribution of receptors for three potential inhibitory neurotransmitters in the brainstem auditory nuclei of the cat. J Comp Neurol 275:288-308.

Grinnell AD (1963) The neurophysiology of audition in bats: temporal parametcrs. J Physiol (Lond) 167:67-96.

Hamill OP, Marty A, Neher E, Sakmann B, Sigworth J (1981) Improved patch-clamp techniques for high-resolution current recording from cell and cell-free membrane patches. Pflügers Arch 391:85-100.

Haplea S, Covey E, Casseday JH (1994) Frequency tuning and response latencies at three levels in the brainstem of the echolocating bat, Eptesicus fuscus. J Comp Physiol [A] 174:671-683.

Hsu S, Raine L, Ganger H (1981) The use of avidin-biotin-peroxidase complex $(A B C)$ in immunoperoxidase techniques: a comparison between $\mathrm{ABC}$ and unlabeled antibody (PAP) procedures. J Histochem Cytochem 29:577-580.

Jagadeesh B, Gray CM, Ferster D (1992) Visually evoked oscillations of membrane potential in cells of cat visual cortex. Science 257:552-554.

Jagadeesh B, Wheat HS, Ferster D (1993) Linearity of summation of synaptic potentials underlying direction selectivity in simple cclls of the cat visual cortex. Science 262:1901-1904.
Johnson BR (1993) GABAergic and glycinergic inhibition in the central nucleus of the inferior colliculus of the big brown bat. $\mathrm{PhD}$ dissertation, Duke University.

Kuwada S, Yin TCT, Haberly LB, Wickesberg RE (1980) Binaural interactions in the cat inferior colliculus: physiology and anatomy. In: Psychophysical, physiological and hehavioral studies in hearing (Van den Brink G, Bilsen FA, eds), pp 401-411. Delft: Delft UP.

Marty A, Neher E (1983) Tight-seal whole-cell recording. In: Singlechannel recording (Sakmann B, Ncher E, eds), pp 107-122. New York: Plenum.

Moiseff A (1985) Intracellular recordings from owl inferior colliculus. Soc Neurosci Abstr 11:735.

Nelson PG, Erulkar SD (1963) Synaptic mechanisms of excitation and inhibition in the central auditory pathway. $J$ Neurophysiol 26:908-923.

Park TJ, Pollak GD (1993a) GABA shapes sensitivity to interaural disparities in the mustache bat's inferior colliculus: implications for sound localization. J Neurosci 13:2050-2067.

Park TJ, Pollak GD (1993b) GABA shapes a topographic organization of response latency in the mustache bat's inferior colliculus. J Neurosci 13:5172-5187.

Pollak GD, Park TJ (1993) The effects of GABAergic inhibition on monaural response properties of neurons in the mustache bat's inferior colliculus. Hear Res 23:115-121.

Roberts RC, Ribak CE (1987) GABAergic neurons and axon terminals in the brainstem auditory nuclei of the gerbil. $J$ Comp Neurol 258:267-280.

Rose JE, Greenwood DD, Goldberg JM, Hind JE (1963) Some discharge characteristics of single neurons in the inferior colliculus of the cat. $I$. Tonotopical organization, relation of spike-counts to tone intensity, and firing pattern of single elements. J Neurophysiol 26:294-320.

Sakmann B, Hamill OP, Burmann J (1982) Activation of chloride channels by putative inhibitory transmitters in spinal cord neurons. Pflügers Arch 392:R19.

Suga N (1964) Recovery cycles and responses to frequency modulated tone pulses in auditory neurons of echo-locating bats. J Physiol (Lond) 175:50-80

Sullivan WE (1982) Possible neural mechanisms of target distance coding in auditory system of the echolocating bat, Myotis lucifugus. J Neurophysiol 48:1033-1047.

Vater M, Habbicht H, Kössl M, Grothe B (1992) The functional role of GABA and glycine in monaural and binaural processing in the inferior colliculus of horseshoe bats. J Comp Physiol [A] 171:541-553.

Winer JA, Larue DT, Pollak GD (1995) GABA and glycine in the central auditory system of the mustache bat: structural substrates for inhibitory neuronal organization. J Comp Neurol 355:317-353.

Yang L, Pollak GD, Resler C (1992) GABAergic circuits sharpen tuning curves and modify response properties in the mustache bat inferior colliculus. J Neurophysiol 68:1760-1774.

Yin TCT (1994) Physiological correlates of the precedence effect and summing localization in the inferior colliculus of the cat. $J$ Neurosci 14:5170-5186. 